\title{
Erratum to: Isolated remethylation disorders: do our treatments benefit patients?
}

\author{
Manuel Schiff • Jean-François Benoist • Bogdana Tilea • \\ Nicolas Royer • Stéphane Giraudier • \\ Hélène Ogier de Baulny \\ Published online: 27 September 2011 \\ (C) SSIEM and Springer 2011

\section{Erratum to: J Inherit Metab Dis}

\section{DOI 10.1007/s10545-010-9120-8}

In the original published article, heading "Discussion", subheading "Available treatment", section 5, there is a mistake in the sentence "With oral methionine supplementation (40$50 \mathrm{mg} / \mathrm{kg}$ per day) ....". It should read:

"With oral methionine supplementation (40-50 $\mathbf{~ m g}$ per day) ...."

The daily dose in mg per $\mathrm{kg}$ is the mistake.

The online version of the original article can be found at http://dx.doi. org/10.1007/s10545-010-9120-8.

\footnotetext{
M. Schiff $\cdot$ J.-F. Benoist $\cdot$ N. Royer

Reference Center for Metabolic Disease,

Robert Debré University Hospital, APHP,

Paris, France

M. Schiff $\cdot$ H. O. de Baulny

Pediatric Neurology \& Metabolic disease,

Robert Debré University Hospital, APHP,

Paris, France

N. Royer

Biochemistry, Robert Debré University Hospital, APHP,

Paris, France

B. Tilea

Pediatric Radiology, Robert Debré University Hospital, APHP,

Paris, France

S. Giraudier

Haematology Laboratory, Henri Mondor University Hospital,

AP-HP,

Créteil, France

M. Schiff $(\bowtie)$

Service de Neuropédiatrie \& Maladies Métaboliques, Centre de, Référence Maladies Métaboliques, Hôpital Robert Debré,

48, Boulevard Sérurier,

75019 Paris, France

e-mail: manuel.schiff@rdb.aphp.fr
} 